\title{
Arm retraction and escape transition in semi-flexible star polymer under cylindrical confinement
}

\author{
Dušan Račko ${ }^{1,2,3} \cdot$ Peter Cifra $^{1}$
}

Received: 10 March 2015 / Accepted: 15 June 2015 / Published online: 4 July 2015

(C) Springer-Verlag Berlin Heidelberg 2015

\begin{abstract}
We studied the structure and dynamics of starshaped polymers by means of coarse-grained molecular dynamics simulations and analysis of structural transitions of semi-flexible macromolecules confined in nano-channels. The conformation of star arms in narrow channels is given by the channel width, arm flexibility and number of arms aligned together in the given region along the channel. We focused on the conformation transition, where all arms are initially stretched in one direction of the narrow channel and were interested in the process of how individual arms escape into a free volume region of channel. We found that the escape transition does not proceed from arm ends but progresses by extension of a loop starting from the branch point; the arms escape in individual steps and the extension of arms depends on how many arms align in parallel in the channel.
\end{abstract}

Keywords Semi-flexible $\cdot$ DNA $\cdot$ Molecular dynamics · Confined $\cdot$ Nano-channel $\cdot$ Electrophoresis

Electronic supplementary material The online version of this article (doi:10.1007/s00894-015-2735-9) contains supplementary material, which is available to authorized users.

Dušan Račko

dusan.racko@savba.sk

1 Polymer Institute, Slovak Academy of Sciences, Dúbravská cesta 9, 84541 Bratislava, Slovakia

2 Center for Integrative Genomics, University of Lausanne, 1015 Lausanne, Switzerland

3 SIB Swiss Institute of Bioinformatics, 1015 Lausanne, Switzerland

\section{Introduction}

Macromolecular confinement is a common effect that occurs in the field of natural and synthetic polymers and processes they are involved in. However, the complex interplay in macromolecular confinement is not yet understood in full detail. Confinement can now be studied with new experimental techniques, like uni-molecular experiments, lab-on-chip experiments using nano-fluidic devices [1-3], DNA separation [4, 5], atomic force microscopy (AFM) [6], etc. Separation and characterization techniques for polymers involve mainly chromatography [7] and ultrafiltration [8, 9]. In molecular biology, confinement plays role in processes like protein translocation [10-12], chromosome segregation [13-17] and protein synthesis [18]. Molecular simulations provide unprecedented insight and complementary information to novel experiments, also for testing and developing new theories. The behavior of linear polymers has been the subject of intense computational exploration [19]; however, despite all this computational and theoretical effort, little is known on polymers with varied stiffness and topology. The problem of molecular stiffness becomes much more interesting under the conditions of confinement where it is no longer only just a matter of different scales but also of mutual relationships between the overall polymer coil size, the persistence length of the chain and the extent of confinement.

In addition to the aspect of the molecular stiffness, molecular architecture may also play an important role in determining molecular properties. Star polymers have unique properties. They represent an intermediate situation between soft colloids and polymers $[20,21]$ and their application ranges from lubrication [22], colloidal stabilization [23], polymer films through drug delivery systems [24-26], contact lenses in medicine [27] or nano-particles in material science $[28,29]$. Recently, star-shaped DNA 
molecules have also been synthesized and their properties have been studied [30, 31].

In the literature some focus has been dedicated to computer simulations of star-shaped polymers under confinement. Dynamic behavior and structure of star-shaped polymers in a slit under conditions of good solvent as well as in a slit with attractive walls have been simulated [32-34]. The case of a linear polymer was presented in studies of chromosome segregation of simple bacteria where a branched-like structure can be identified during certain phase of replication $[14,35]$. Interpretations important for star-shape-like behavior were pointed out also within the free energy studies of condensed system of linear chains [36]. Very recently, an equilibrium distribution of the arms of a 3-arm polymer in a cylindrical confinement was explored for the first time by means of computer simulations [37], and a case of a pressurized molecule under the tip of an AFM probe has been simulated [38].

Studies to date have not paid detailed attention to the aspect of molecular stiffness, and no work has yet focused on the problem of a semi-flexible star polymer in cylindrical confinement. In this contribution, we performed a computer simulation study of the dynamic behavior and topological properties of a 4-arm star polymer including the aspect of chain stiffness. We focus on a cylindrical confinement with different diameters determining various confinement strengths. In the initial conformation, the polymer arms are aligned so that the arm ends lie at one side and the connecting point is located on the opposite side. This situation simulates the case of a star polymer driven to a nano-pore, when it is thought that it will enter the tube by its center of mass [39]. The initial configuration of the molecule as well as its dynamic movements during the escape transition of individual arms resemble an octopus escaping through a small hole [40]. Here, the arm transition will be examined from various aspects, such as the evolution of molecular conformations from molecular dynamics (MD) simulations and theoretical free energy calculations, while addressing also some experimental observations. This work is a continuation of our related study on segregation of semiflexible polymer chains in the tube-like confinement [35].

\section{Methods}

The star polymer molecule was represented by a simplified bead-spring model. The star was built from two chains of 201 beads each, connected in the middle by a bond. The spacing between beads in the initial configuration corresponds to the average bond length $\langle l>=0.965$ [35]. The size of each of the arms without branch point is thus $N=100$ beads. In the initial conformation, the four arms of the molecule are aligned so that it resembles an octopus - the branch point is at one end and the ends of arms (tentacles) are on the opposite side along the channel. This conformation represents a case of a molecule in the electrophoretic medium when the electrostatic field is turned off and the molecule starts to freely relax. The molecule is confined in two directions in an infinite cylinder of diameter D.

The potential function for bonded and non-bonded interactions comprises three terms. The excluded-volume interactions between both bonded and non-bonded beads were modeled by a fully repulsive shifted and cut potential (Weeks-Chandler-Andersen) [41], so that for $r<\sqrt[6]{2} \sigma ; U=$ $U_{\mathrm{WCA}}(r)=4 \varepsilon\left[(\sigma / r)^{12}-(\sigma / r)^{6}+0.25\right]$ and $U=0$ otherwise. Conventionally, $r$ denotes the center-to-center distance between two beads and $\sigma$ represents the bead diameter. We use $\sigma=1$ and it is identified with a length unit for numerical values as indicated below. The same model equation is used to describe interactions between the beads and the wall of the confining cylinder.

Within the bonded interactions, besides bond stretching, variable bending angle is also included in order to simulate the stiffness of the macromolecular backbone. The bond stretching is modeled by the Finite Extensible Non-linear Elastic potential, $U(r)=-0.5 K r_{\mathrm{o}}^{2} \ln \left(1-r^{2} / r_{\mathrm{o}}^{2}\right)$, where $K=30 \varepsilon /$ $\sigma^{2}$ and the maximum bond length is $r_{\mathrm{o}}=1.5 \sigma$. The energetic penalty for angle bending is expressed by a cosine bond angle potential of two consecutive bonds forming an angle $\phi$, $U_{\mathrm{b}}=K_{\mathrm{b}}(1-\cos \phi)$, where the bending constant $b=P /\langle l\rangle=K_{\mathrm{b}} /$ $k_{\mathrm{B}} T$ is a measure of the chain stiffness related to the persistence length $P$, where the simulated average bond length in a chain is $\langle l>=0.965 \pm 0.023$. In order to represent the dsDNA chain in a physiological buffer by this model we can set $\sigma=$ $2.5 \mathrm{~nm}$ and $b=20$ that represents a common value $P=50 \mathrm{~nm}$ [48]. Note that the potential functions apply also to the branch point in the same way as for the whole molecule, including the bond stretching, bending and intermolecular forces.

By using these force-field settings and starting from initial configuration, the MD behavior was simulated by means of the Langevin approach. The settings for the thermostat and velocity integrator were taken from related computational simulations [42-44]. Langevin dynamics attempts to extend classical MD for the effects of friction and velocity perturbations that may occur in the presence of a solvent. The movement of polymeric beads is described by stochastic differential equations as $m \ddot{\mathbf{r}}_{I}=-\nabla U\left(\mathbf{r}_{I}\right)-\gamma m \dot{\mathbf{r}}_{I}+\sqrt{2 \gamma k_{\mathrm{B}} T m} R(t)$, where $\nabla U$ is the potential for the bead interaction, $\gamma m \dot{r}_{I}=\xi v_{i}$ expresses the friction $(\gamma=\xi / m=0.5)$ and $\sqrt{2 \gamma k_{\mathrm{B}} T m} R(t)=\mathbf{F}_{I}^{R}$ is the random force. Langevin MD simulations were performed in the Extensible Simulation Package for Research on Soft Matter (ESPResSo v. 3.01) [45]. The equations of motions were integrated by means of velocity Verlet integrator with a given time step $\Delta \tau=0.005 \tau_{\mathrm{o}}$, where the time unit is specified by the chosen parameters as $\tau_{o}=\sigma \sqrt{m / \varepsilon}=1$. For a $2.5 \mathrm{~nm}$ segment of DNA, the Lennard-Jones (LJ) time 
unit thus corresponds to $2.1 \mathrm{~ns}$. The simulations were carried out using constant number of beads, volume and temperature. The temperature was controlled by means of Langevin thermostat and we chose the temperature such that $k_{\mathrm{B}} T / \varepsilon=1$.

In order to investigate the structural transitions and mutual interplay between molecular stiffness, confinement strength and their effect on dynamics of conformational transition, MD runs were performed for three bending parameters, $b=$ 0,5 and 20, and several cylinder widths $D=5,6,8,10$ and 20 for the stiffest molecules. The lowest $D$ was determined from earlier work and testing [35]. Below $D=5$, the molecule gets stuck in the channel with the arrest of dynamics.

Molecular dynamics simulations were undertaken in two stages. At first, the thermal equilibration took place when the system was warmed up during 10,000 MD steps $(\tau=500)$. Next, an MD production run started during which data for analyses were collected. The length of simulations was chosen so that the transition effects of interest were exposed. The simulations took $2 \times 10^{8} \mathrm{MD}$ steps $\left(\tau=10^{6} \sim 2.1 \mathrm{~ms}\right)$. In the case of fully flexible chains, the transition process was observed to be faster; thus, in order to investigate the dynamics, shorter simulations with higher frequency of storing coordinates were performed $\left[3 \times 10^{6} \mathrm{MD}\right.$ steps $\left.\left(\tau=10^{4}\right)\right]$.

For the purposes of tracking the transition effects, several topological descriptors were defined (Fig. 1) and their instantaneous values were evaluated along the trajectory. The span of the molecule naturally comes to mind as the first descriptor of large-scale conformational changes. This is calculated as the largest distance between two beads that can be found on the molecule $R_{\mathrm{sp}}=\left\langle\operatorname{MAX} d\left(\mathbf{r}_{I}, \mathbf{r}_{j}\right)\right\rangle ; I, j=1, \ldots, N$. The span of each arm was calculated in a similar manner, with the maximum distance between two beads forming an arm being evaluated. A complementary descriptor was defined as the angle between arms, or the major axes of inertia computed for particular arms. First, the head of the octopus was shifted to the origin so that $\hat{\mathbf{r}}_{I j}=\mathbf{r}_{I j}-\mathbf{r}_{0}$, where $\mathbf{r}_{i j}$ is a position vector of bead $I$ on $\operatorname{arm} j$.

The gyration vector of instantaneous coil shape for that particular arm was then calculated from the longest component of three principal components of radius of gyration of this arm [46]. The angles between all these arm vectors were computed; hence, we obtained six angles $\theta$, the widest of which (the maximum angle) was used to indicate the extent of arm transition. The ambiguity between flat and full angles during evaluation of eigenvectors was checked by the position vectors of the arms' center of mass along the trajectory. This is the parameter with the highest sensitivity to escape transition. Finally, we also used a range of arms defined as the maximum span of the molecule with respect to the position of the branch point, where the positive or negative signs denote the position with respect to the branch point of the star (Fig. 1).

\section{Results and discussion}

\section{Arm transition from maximum span and angle}

When a star-shaped molecule is driven into a pore or through electrophoretic media (gel or resin), the star molecules are known to move in a squid-like manner [47]. Once the molecules are squeezed in a channel, they form a U-shape conformation along the channel axis [47]. In our simulations, we started accordingly from the initial conformation in which the arms of molecule were aligned together and stretched at one side along the channel ( $4-0$ conformation), and then the molecule was relaxed within the MD simulation. This represents the case when the molecule is already inside a pore and the dragging force is turned off.

At first, in order to reduce number of parameters, we evaluated the behavior of fully flexible molecules without considering chain stiffness (zero bending penalty in the model $b=0$ ). The evolution of maximum span along the trajectory on Fig. 2 a shows that the molecule at first shrinks from the initial extended linear conformation in a very fast process in order to reach a coil shape. The molecular span then starts to rise and this indicates translocation of an arm to the opposite direction towards the free part of the channel. Figure 2a evaluates the additional effect of diameter of the channel. We can observe that the process is faster for stronger confinements and that equilibrium is reached sooner in a narrower channel, as the local concentration is the driving force for the process. After equilibrium is reached, the maximum span oscillates around a constant value. In wider channels, the maximum span of the equilibrium conformation is smaller as the lower confinement allows the molecule to decrease its gyration radius [35].

Drawings in the figure represent typical conformations of the molecule at each phase of the escape transition obtained based on molecular visualization of the MD trajectories. As we observed, the process of escaping an arm is a stepwise process and always occurs with one arm side by side with another. In the case of fully flexible chains, both arms were observed to translocate and hence the fully equilibrated arms form a 2-2 conformation. The equilibration of arms is consistent with the recent simulations of a 3-armed polymer in cylindrical confinement. However, the 3 -arm polymer represents another specific case when the odd arm redistributes randomly between the two oppositely stretched arms and remains only partially translocated [37].

Furthermore, the maximum angle found between arms was evaluated (Fig. 2b). This topological parameter gives complementary information to the molecular span and is very sensitive for detecting the transition. At first, in the period when the arms are aligned together at one side along the channel, the value of all angles is close to zero. When the arm escapes to the opposite side of the channel, the angle reaches a value of 180 angular degrees. The evaluation of the maximum angle 
Fig. 1 Scheme of topological descriptors used to evaluate the escape transition; the picture shows an actual snapshot from the molecular dynamics (MD) trajectory

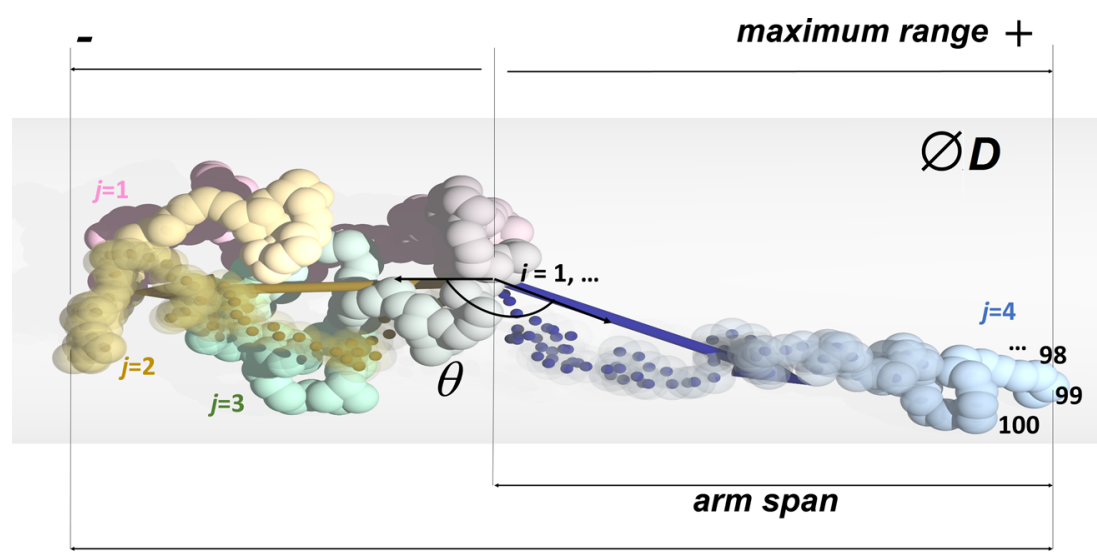

Maximum span

shows a sharp change, indicating the escape transition. This infers that the process of transition is rather fast for flexible arms and follows the dependence on the channel diameter discussed with the maximum span of molecule. Figure 3 shows the evolution of the maximum span of individual arms

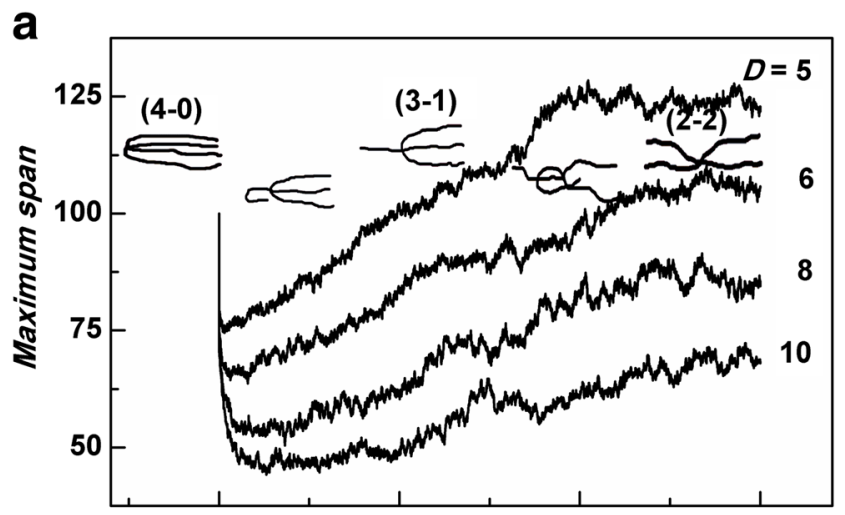

b

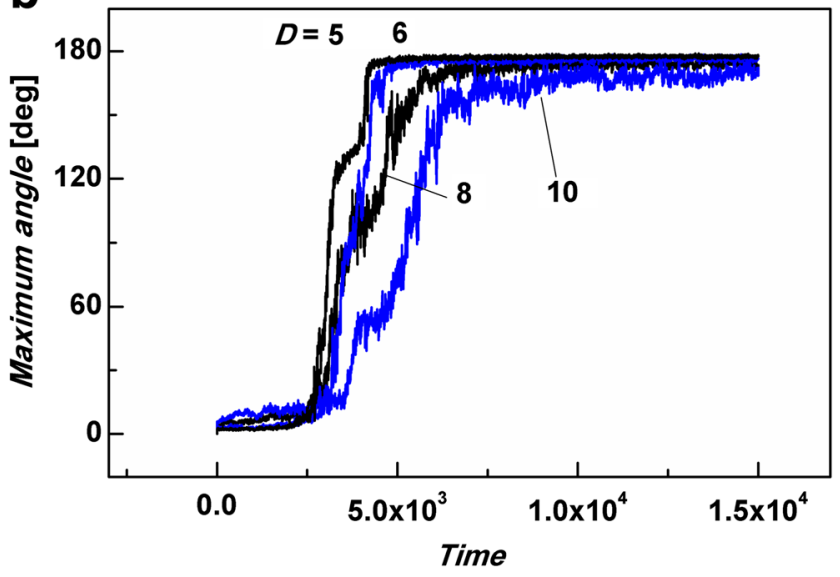

Fig. 2a,b Escape transition of fully flexible 4-arm polymer. a Maximum span in units of $\sigma$ and $\mathbf{b}$ maximum angle between arms found on the star polymer obtained as an average of five runs at different strengths of confinement indicated by a diameter of the confining channel, $D$. Drawings indicate typical conformation of the molecule at each particular phase of transition as compared to the maximum span of the whole molecule. The escaping arm (red line) can be identified as the arm that reaches the lowest span during the period where it first forms a shoulder and then crosses to the opposite side of the channel. When the arm escapes, it stretches again and the span oscillates randomly around an equilibrium value similar to that of the other arms.

In subsequent $\mathrm{MD}$ runs, the complexity of the system was increased and the influence of the molecular stiffness on the escape transition was investigated. Figure 4a shows the topological parameters evaluated along with the MD trajectory. The escape transition was also observed in this case; however, the process was slower with increasing molecular stiffness.

In order to observe the full transition, much longer MD runs were needed as compared to fully flexible molecules. The molecular stiffness, which induces stretching of parallel extended arms, causes the local concentration along the channel to decrease so that arms will not feel each other, thus the driving force for arm escape is lower. At the same time, the

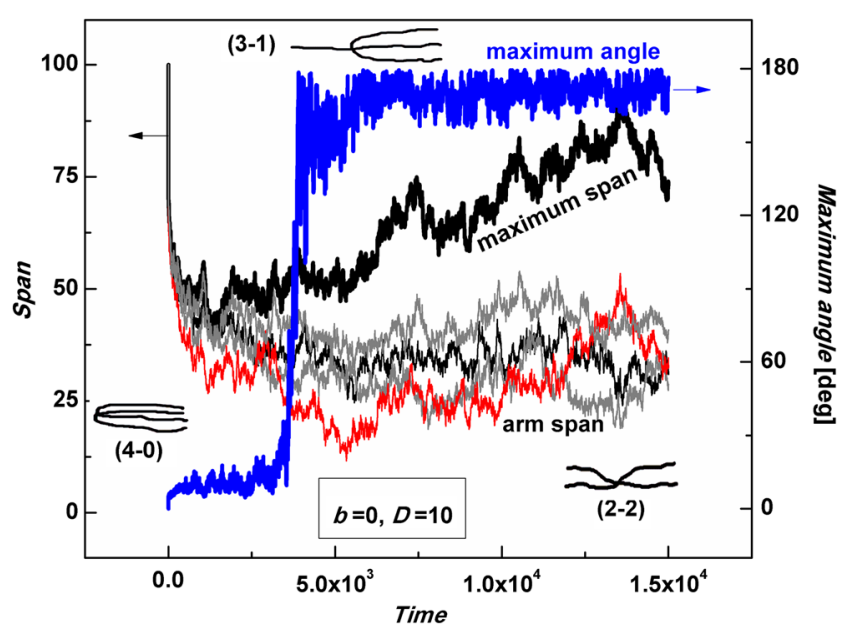

Fig. 3 Escape transition and span of individual arms compared to the maximum span $[\sigma]$ of the molecule. The maximum angle between arms is also shown in order to determine the transition region. The data are from a selected single MD run for molecular stiffness $b=0$ and diameter $D=10 \sigma$ 

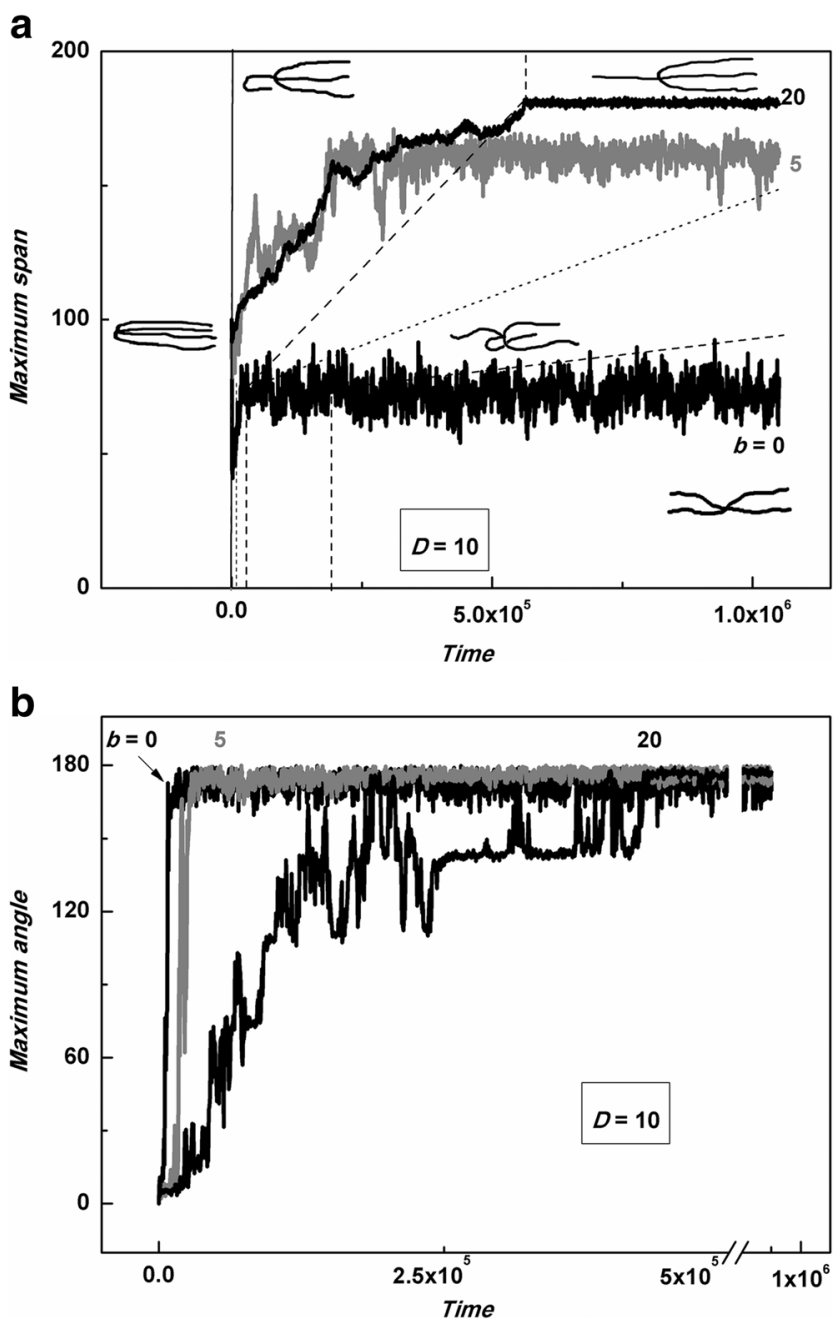

Fig. 4 Escape transition and topological parameters evaluated along the $\mathrm{MD}$ trajectory for a maximum span in units of $\sigma$ and $\mathbf{b}$ maximum angle of molecule for different bending parameters $b$ indicated on chart, and channel with diameter $D=10 \sigma$. Dashed lines Borders of conformational states observed from the snapshots of MD trajectories (drawings). The data show averages from 5 runs for each stiffness

energy needed for bending the arm increases with molecular stiffness. Similar behavior has been observed in computational studies of segregation of semi-flexible molecules in a nanochannel [35]. It should be noted here that stiffer molecules also stabilize temporarily in a transient state, forming hairpins [48]. Figure $4 \mathrm{~b}$ shows that hairpins are rather stable structures persisting for half of the simulation run. Moreover, we observe from the snapshot of MD trajectories (not shown here) that the equilibrium conformation $2-2$ is typically not reached in the case of stiff molecules in a narrow channel, hence only one arm passes through (Fig. 4a). Twice longer runs and statistics accumulated during the simulations study additionally confirm this observation. As inferred from Fig. 4a, the maximum span of the molecule equilibrates to larger spans with increasing the polymer stiffness as the stiffer chains are naturally stretched.
Increasing the diameter of the channel decreases the confinement strength; the energy necessary for arm bending is lower as the arm does not have to be bent so much in order to escape from the initial configuration. Figure 5 shows the maximum distance from the branch point, which is centered at the origin (zero). Hence, the range for each individual arm is calculated also with its relative position indicated by a positive or negative sign. As discussed above, in the case of a narrow channel, the transition occurs only for one arm (Fig. 5a). At first it is stabilized in a transient hairpin structure during a certain period. Afterwards, a sharp transition is observed and the star polymer stabilizes in 1-3 conformation of arms.

However, for a wider channel when the channel diameter is the same as the persistence length, $b \cong D$, the second arm follows the first transition and equal distribution of arms (2-2) along the channel is achieved (Fig. 5b). In the case of the

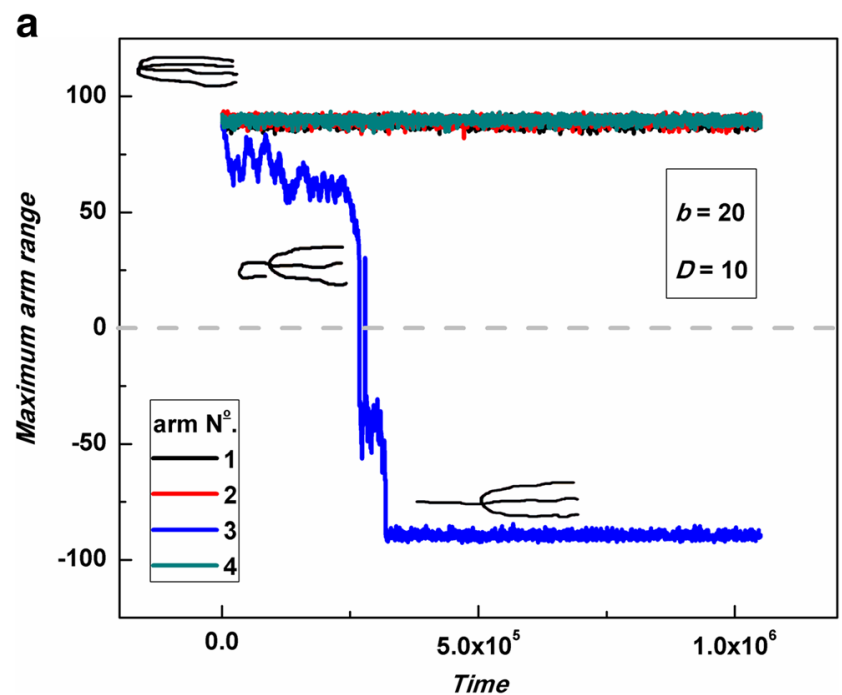

b

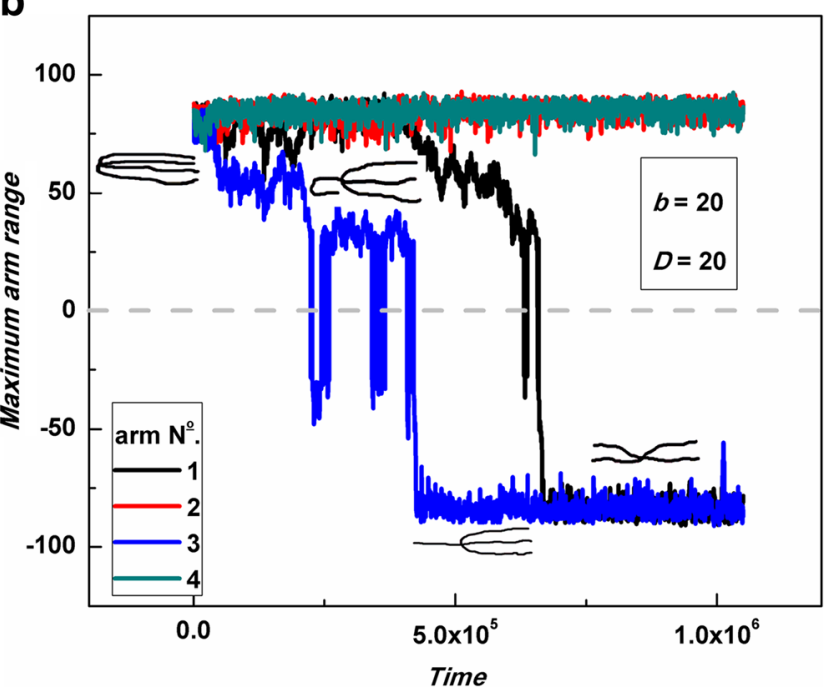

Fig. 5 Maximum range of arms centered on the branch point of the "octopus" in units of $\sigma$ computed for semiflexible molecules with $b=20$ and channel diameters $\mathbf{a} D=10 \sigma$ and $\mathbf{b} D=b=20$ 
second arm, the hairpin structure lasts for less time, as seen from the transition width. We also clearly see that the transition is a stepwise process, with always only one arm passing at a time. As support for these observations, we provide a video as a part of the Supplementary Material.

A similar graphical representation has been used recently in a computer simulation study of a 3 -arm polymer in a cylindrical confinement [37]. In the case of the 3 -arm polymer, the escaping arm was observed to jump randomly from one side to the opposite with respect to the center of the star polymer, with other two arms placed in opposite directions. Such tumbling of the arms was observed also in simulations of 3-arm polymers confined in a slit with attractive walls [33]. No such process was observed here in the case of a star polymer with an even number of arms, or in the case of molecules with flexible arms within a time period exceeding 100 times the time necessary for transition to occur (Fig. 4b).

\section{Confinement free energy as a driving force of arm transition}

The decrease in the molecular span of a channel due to formation of hairpins is relatively well known and explored in the literature [48, 52]. A less well known phenomenon is stretching the size of the arms in the presence of other arms in the channel when they extend along each other. In our recent work on segregation of macromolecules in a cylindrical channel, we observed that the molecules indeed elongate during the period when they mutually "overlap" [35]. The effective diameter of the channel would be $D / \sqrt{2}$ for two [15], $D / \sqrt{3}$, for three, and $D$ $/ \sqrt{4}=D / 2$ for four overlapping parallel arms. This represents a reduced cross-sectional area of the channel to $1 / 2$, $1 / 3$ and $1 / 4$. To apply these changes of effective channel width to arm extension and to the confinement penalty, one has to start from a single confined chain. Although the route according to Flory theory could be used, we apply here the scaling picture, which shows also structural aspects. The single chain of $N$ segments in a channel of diameter $D$ attains a cigar-like shape composed of $\mathrm{N} / \mathrm{g}$ blobs of size $D$, where a chain of $g$ segments in each blob has a character of excluded volume chain. Thus, for a semiflexible chain in a blob, we have $[49,50], D \cong$ $L_{\mathrm{b}}{ }^{3 / 5}(w P)^{1 / 5}$. Here, $w$ is the chain width, assumed to be equal to the segment size $\sigma$, and $P$ is the persistence length of chain. The contour length stored in one blob, $L_{\mathrm{b}}=g w$, is an analogy to the contour length of the chain, $L=N w$. The overall chain extension along the channel is thus

$R_{\mathrm{II}} \cong D\left(\frac{N}{g}\right)=L\left(w P / D^{2}\right)^{1 / 3}$
The free energy of confinement is given by the number of these blobs, each blob contributing by a penalty of $k T$.

$\frac{\Delta A_{c}}{k T} \cong \frac{N}{g}=L(w P)^{1 / 3} D^{-5 / 3}$

If we have two chains that extend along each other in the same region of the channel then each chain (here one of the parallel arms) is placed in an effective tube with adequately reduced diameter. The chain of blobs of smaller effective size $D / \sqrt{ } 2$ is accordingly given as $R_{\mathrm{II}} \cong(D / \sqrt{ } 2) N / g$, where the number of segments $g$ stored in one blob is now given as $D / \sqrt{2} \cong$ $L_{\mathrm{b}}{ }^{3 / 5}(w P)^{1 / 5}$. For a coil extension at full overlap of two coils, we obtain the following relation

$R_{\mathrm{II}} \cong 2^{1 / 3} L\left(w P / D^{2}\right)^{1 / 3}$

The numerical factor here expresses the expansion of coils by $26 \%$ relative to the single coil in the same channel [15]. Using the effective reduced tube diameter for each of two chains also for free energy leads to the following relationship:

$\frac{\Delta A_{c}}{k T} \cong \frac{N}{g}=2^{5 / 6} L(w P)^{1 / 3} D^{-5 / 3}$

This approach to chain extension and free energy of parallel chains in the channel of overlapping coils can be extended to three and four chains (using the effective diameters $D / \sqrt{ } 3$ and $D / 2$ ) in the same region of the channel. This represents the situation of the 4-arms star polymer extending initially all arms in one direction in a narrow channel. As a result, the predicted relative elongation of the arm relative to the single chain situation in channel, Eq. 3, should have a numerical factor $2^{1 / 3}(+26 \%)$ in the case of two overlapping arms $[15], 3^{1 / 3}(+44 \%)$ for three, and $2^{2 / 3}(+59 \%)$ for four aligned arms in the confining channel. In similar vein, for the prefactors in the free energy of each arm given by Eq. 4, we obtain factors $1,2^{5 / 6}, 3^{5 / 6}$ and $2^{5 / 3}$ again for $1,2,3$ and 4 arms, respectively. The theoretical prediction that the chain extension in parallel overlapping chains along the channel is larger than that in the absence of the other chains, as is presented here and originally applied to two chains or a ring [15, 51], was confirmed in an experiment where the extension in folded portions of DNA in channel was approximately $30 \%$ larger than the equilibrium extension of unfolded molecules [52].

These relations for the free energy of a single arm in situations when the arm is alone in a channel of diameter $D$, or aligned along another one, two or three other arms of the same length, can also be used to answer natural questions that arise. The conformational change from initial 4-0 to a 3-1 situation (one arm released from the initial conformation) is given by overall release of free energy per star given by Eq. 4 with a numerical factor $4 \times 2^{5 / 3}-\left(1+3 \times 3^{5 / 6}\right)=4.21$. The release of a second arm, representing a change from a $3-1$ to a $2-2$ 
conformation, is given by a smaller decrease in free energy with the factor $3 \times 3^{5 / 6}+1-4 \times 2^{5 / 6}=1.37$. This prediction is consistent with our observation that relaxation of the second arm is limited, i.e., not forbidden but observed typically only for flexible chains (Fig. 2). By analogy, the largest decrease in free energy (numerical factor 5.57) represents the conformational change from 4 to 0 to symmetric conformation 2-2. Similarly, one can also understand the free energy change in the process of arm relaxation by looping from branching point into the free volume side of the channel or, alternatively, by retraction of the arm from the chain end, in which one of the arms backfolds along the others and thus enters a region of higher free energy formed by the overlap of 5 arm sequences for a certain time until it is fully relaxed to the greater free volume side of the channel. The latter process is very prohibitive relative to looping (hernia) from a branch point into the free volume part of the channel.

In order to evaluate the elongation and to test this approach, we evaluated arm average spans in particular periods when a given number of arms was aligned in the channel. The periods for evaluating spans and averaging started after an initial equilibration period during which the arms were still aligned together. In order to eliminate the effect of the transitional arm on the average length of the aligned arms, the transiting arm was excluded from averaging. The parameters of two aligned arms were obtained from all four arms in equilibrium $\mathrm{N}$ conformation. Figure 6 shows the averages of the arm span computed as a function of the number of parallel arms, and as a function of channel diameter for flexible as well as for semiflexible molecules. The number of parallel arms in the overlap has an evident effect on arm span for fully flexible molecules. The size of the arm increases in the presence of more parallel arms. The effect fades out with increasing diameter of the channel as the confinement strength decreases and the conformational space opens.

The inset in the chart shows a comparison of the computed relative elongations reduced by the value in the single arm situation with the theoretical predictions given above. Although the trend is preserved, the relative elongations from simulations are slightly lower. The reason for this might be that the chains are joined into a star and do not represent individual confined linear macromolecules. Before the onset of transition occurs, there is a period when all arms are equally probable to enter transition, which may influence the molecular span during averaging and cause apparent shrinking of the molecule; this was seen also in comparison to theoretical predictions. In the case of stiff molecules, elongation was not observed, irrespective of the number of overlapping arms in the channel, and consistent with what was observed in our earlier work on chain segregation in channels [35]. The stiff molecules are stretched in the channel and influence each other less due to the lower local concentration. Thus, the driving force for the escape transition is also less affected by the

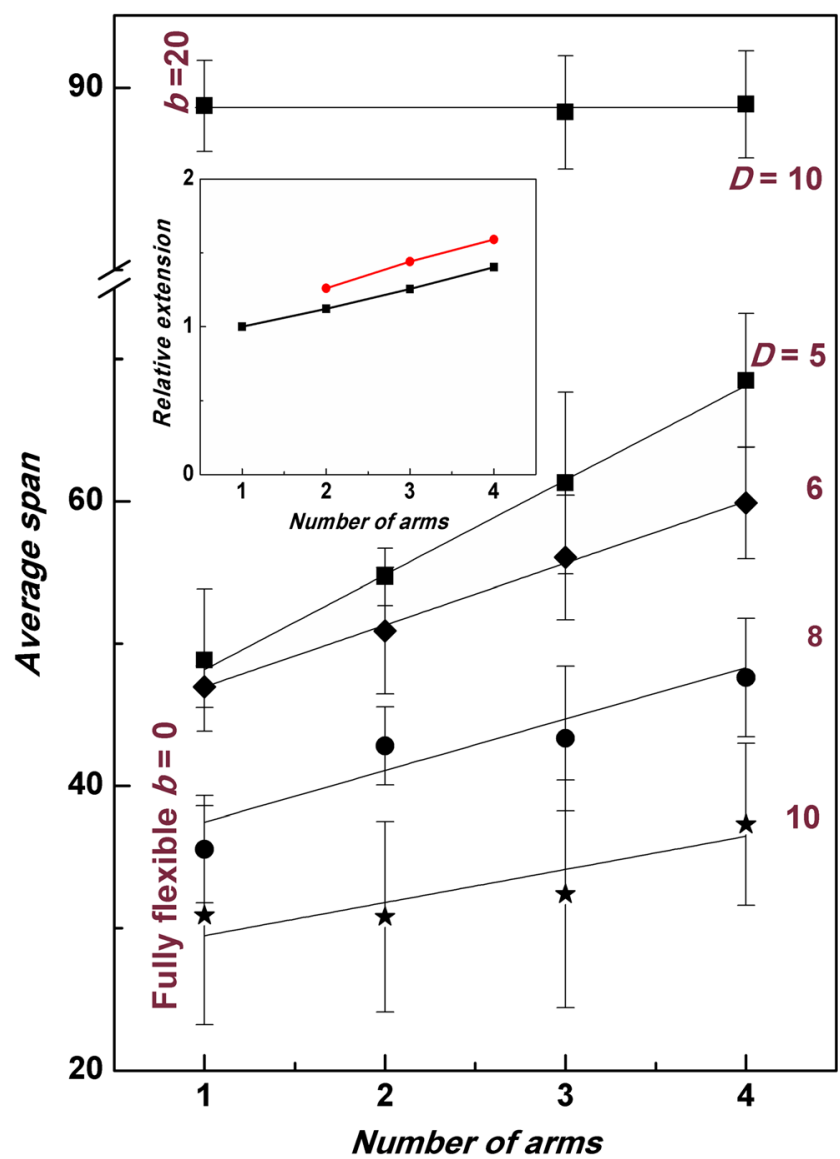

Fig. 6 Mutual influencing of the arms evaluated by arm span for given channel radii in units of $\sigma$. The $x$-axis indicates the number of aligned arms. Inset Comparison of arm extension in parallel arms reduced by extension in single arm situation (घ) with the theoretical predictions for flexible chain in the narrowest channel $(\bullet)$

number of overlapping arms. This is also one of the reasons for observation of stable 1-3 distribution of arms in narrow channels for stiffer chains and no transition to a 2-2 conformation (see Fig. 4). In addition, one should note that the trend of free energy penalty with the chain stiffness for strong confinement (Odijk regime) is reversed in comparison to Eq. 4 for moderate confinement in the blob regime and that stiffer macromolecules experience a lower confinement penalty [53]

$\frac{\Delta A_{c}}{k T} \cong L P^{-1 / 3} D^{-2 / 3}$

\section{Arm transition mechanism from molecular motions}

Next, we analyzed the motions of the arm during transition. In the classical notion by de Gennes [54] the relaxation of the star shape polymer in a melt of stars or crowded environment should start from chains' ends by retracting the arms. It is also thought that inner segments closer to the connecting point of the star would relax much slower than the outermost ones. As yet it is still unclear if the motion in a tube starts from its ends 
or by forming a shoulder [55]. Testing the arm retraction mechanism in fixed channel here was the key point of the present study. In order to test this mechanism, the beads forming the arms were indexed starting from the branch point $(I=0)$ of the molecule with $I=1$ to $I=100$ for the end segment of each arm. Next, the maximum span between the most distant beads was evaluated, and the indexes of the most distant beads were monitored for the corresponding time in the MD trajectory.

Figure 7a shows the evolution of indexes of the most distant beads along the trajectory. To begin with, the indexes of the most distant beads were $I=1$ and $I=100$, because in the initial configuration the arms were aligned on one side and the connecting point on the other side. If the escaping proceeded by the arm's end there would indeed be a jump-like behavior on the graph, showing sudden behavior of the most distant beads both with the index of 100 after a relatively long induction period, as shown by the dashed line in Fig. $7 \mathrm{~b}$. Surprisingly (in comparison to expectations for the situation in melting stars), the lower index number increases continuously, providing numerical evidence of a shoulder (loop) formation and subsequent unwinding of the polymer arm. This was observed also by visualization of MD trajectories and this is also in accord with the confinement of free energy of the multiple parallel arms presented above (the loop proceeds directly to the free volume region while relaxation from the arm end proceeds through a narrower space along multiple parallel arms). The mechanism of escape transition is the same for fully flexible as well as for semiflexible macromolecules. In the case of fully flexible molecules, the escape is faster and, as we can see, the arm ends are not always the most distant part of the molecule (Fig. 7a) as the result of molecular flexibility that allows wiggling chain ends in a tail-like fashion. This is the only difference in the arm movements during the escape transition from the semi-flexible molecules, which remain straightened so that the beads at the arm ends always remain one of the most distant beads (Fig. 7b). The movement of the arms during the escape transition resembles the motion of an
Fig. 7 Indexes of the most distant beads during the transition. The chain is indexed from the connecting point as $I=1$ to the chain's end with $I=100$ as computed for a fully flexible molecules and $\mathbf{b}$ semiflexible molecules with $b=20$, both in a narrow channel with $D=10$. Dashed line Process of arm retraction if it were to proceed by arm end (opposite to what is observed)
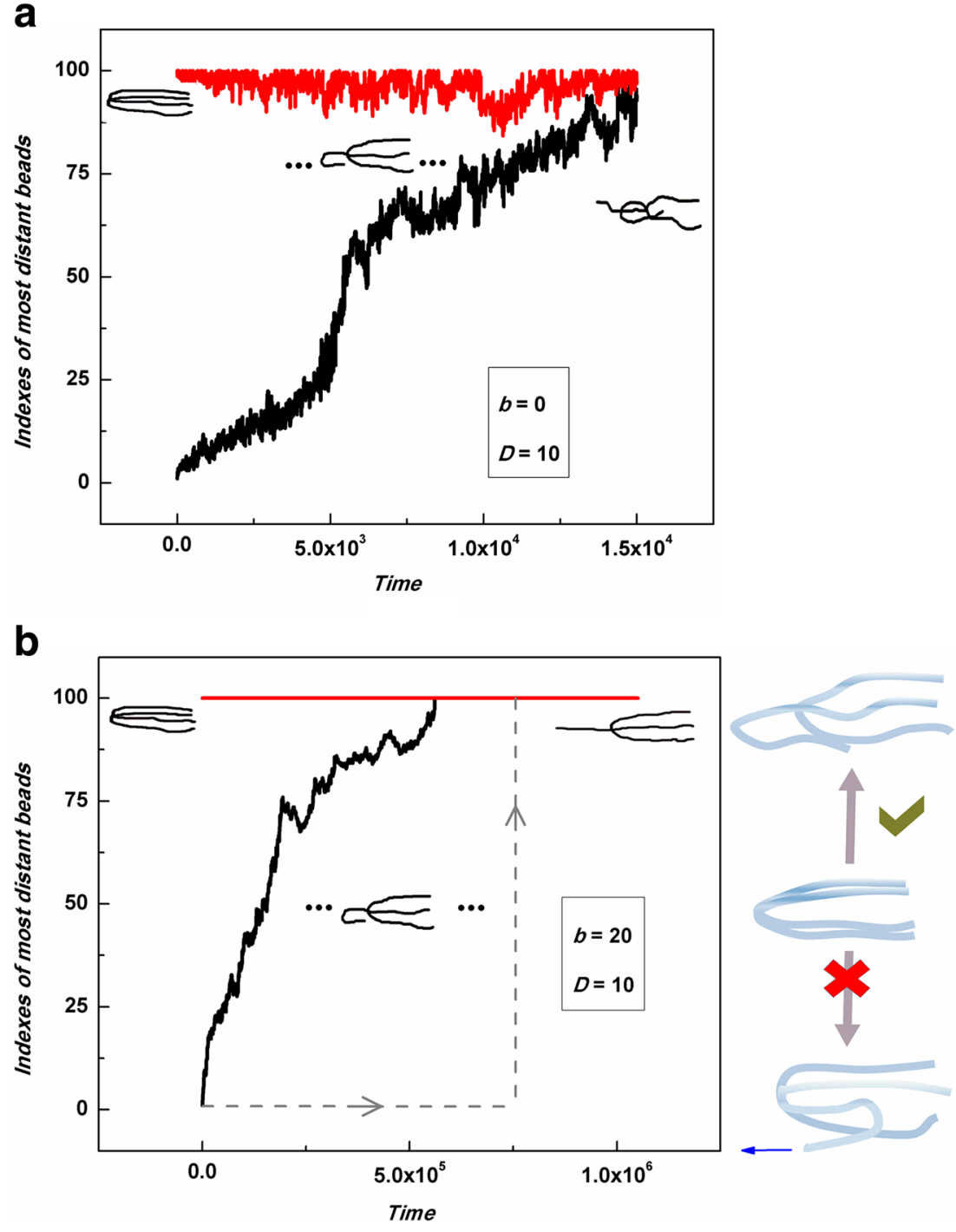
octopus escaping through a narrow hole by loop formation at the head and extension through the hole [40].

Similar dynamic processes as this relaxation of arms were recently interpreted in terms of transition theory [56], which is based on the energy barrier involved in the transition. For instance, in the segregation of macromolecules in a nanochannel [57], the transition barrier considered leads to an induction period during segregation. However, the presence of such a barrier is still a matter of discussion. Polson et al. [58] directly estimated free energy in the segregation process from simulation, which was observed without the barrier. It should be noted that the arm relaxation process here is spontaneous, whereas arm relaxation in a confined star proceeds along the decrease of confinement free energy. In particular, arm relaxation from star head by loop extension into the free volume side of the channel is such a process, while arm relaxation from the arm end would proceed across the energy barrier as described above in confinement free energies for different numbers of parallel arms.

\section{Conclusions}

We performed MD simulation of fully flexible and semiflexible star polymer with four arms confined in a nanotube with various diameters. The initial configuration of the molecule represents a case of a star polymer placed into a confining cylinder by a dragging force, with all arms extended to the same side along the channel. The MD runs were performed in order to relax the molecule.

Within the relaxation, escape transition behavior was observed. Escape transition was faster for fully flexible molecules and slowed down with increasing backbone stiffness. In the case of fully flexible molecules, an even distribution of arms along the channel was achieved. For semi-flexible molecules under strong confinement, only one arm was able to pass through with equilibration to a 1-3 conformation. Uneven distribution is caused by the fact that (1) the stiffer molecules remain straightened so that the local concentration - the driving force for the transition - is weakly affected by the number of coexisting arms in a channel, and (2) the energy for bending the arm increases with the molecular stiffness. We presented a simple scaling picture of arm extension and the free energy penalty of confinement of arms depending on the number of parallel arms. This can be used for prediction of release of confinement penalty on arm relaxation in a channel and is in accord with the simulation results.

Effects of confinement on molecular structure of the arms were evaluated. In the case of the fully flexible molecules, the span of the arms depended on the number of mutually aligned parallel arms. The larger extension with more parallel arms is in qualitative agreement with the suggested prediction for nano-channel. The effect fades out with increasing width of the channel. In the case of semi-flexible molecules, the relative elongation observed was not in agreement with our previous study on segregation of parallel chains in channel [35]. On the other hand the semi-flexible molecules were observed to create a transient hairpin-like structure. These persist for longer with greater molecular stiffness.

Finally, we contribute to the discussion on motions and mechanism during arm retraction. In order to specify the mechanism of arm retraction we designed a method that traces which part of the chain is responsible for the relaxation. We observed that the escape transition in the channel starts from the branch point of the star polymer, smoothly forming a shoulder, and proceeding by straightening the looped arm to the free end of the channel. This resembles the movements of an octopus escaping through a narrow hole. This situation of a star polymer in a fixed channel is somewhat different from the situation in a melt of stars with the arms in soft transient tubes. Nevertheless, we think that these findings have implications for arm retraction in different situations of confined or crowded systems involving star polymers. Moreover, the indexing approach, which was helpful in establishing the mechanism here, can be adapted to the melt of stars. After proper modification, it will also provide specification of details of arm retraction in melt of stars.

Acknowledgments This work was supported by the Slovak Research and Development Agency (Grant No. SRDA-0451-11) and the Scientific grant agency of the Ministry of Education of the Slovak Republic (VEGA Grant Nos. 2/0093/12 and 2/0068/13).

\section{References}

1. Reisner WW, Beech JP, Larsen NB, Flyvbjerg H, Kristensen A, Tegenfeldt JO (2007) Phys Rev Lett 99:058302

2. Reisner WW, Morton KJ, Riehn R, Wang YM, Yu Z, Rosen M, Sturm JC, Chou SY, Frey E, Austin RH (2005) Phys Rev Lett 94: 196101

3. Tegenfeldt JO, Prinz C, Cao H, Chou S, Reisner WW, Riehn R, Wang YM, Cox EC, Sturm JC, Silberzan P, Austin RH (2004) Proc Natl Acad Sci USA 101:10979

4. Han J, Craighead HG (2000) Science 288:1026-1029

5. Matsuoka T, Kim BC, Huang J, Douville NJ, Thouless MD, Takayama S (2012) Nano Lett 12(12):6480-4

6. Sevick EM (2000) Macromolecules 33:5743-5746

7. Wang Y, Masur A, Zhu Y, Ziebarth J (2010) J Chromatogr A 1217: 6102-6109

8. Jin F, Wu C (2006) Phys Rev Lett 96:237801

9. Hong LZ, Jin F, Li JF, Lu YJ, Wu C (2008) Macromolecules 41: 8220

10. Muthukumar M (2001) Phys Rev Lett 86:3188

11. Muthukumar M (2003) J Chem Phys 118:5174

12. Kumar R, Muthukumar M (2009) J Chem Phys 131:194903

13. Jun S, Thirumalai D, Ha BY (2008) Phys Rev E 101:138101

14. Jun S, Arnold A, Ha BY (2007) Phys Rev Lett 98:128303

15. Jung Y, Jeon C, Kim J, Jeong H, Jun S, Ha BY (2012) Soft Matter 8(7):2095-2102

16. Jun S (2006) Proc Natl Acad Sci USA 103:12388 
17. Arnold A, Jun S (2007) Phys Rev E 76:031901

18. Lu J, Deutsch C (2005) Nat Struct Mol Biol 12:1123

19. Polymers in Confined Environments, Granick S, Binder K, de Gennes P-G, Giannelis EP, Grest GS, Hervet H, Krishnamoorti R, Léger L, Manias E, Raphaël E, Wang S-Q (1999) Adv Polym Sci 138

20. Willner L, Jucknischke O, Richter D, Farago B, Fetters L, Huang JS (1986) Europhys Lett 2:137

21. Semenov AN, Vlassopolous D, Fytas G, Vlachos G, Fleischer G, Roovers J (1999) Langmuir 15:358

22. McLeisch TCB (2002) Adv Phys 51:1379

23. Eisenriegler E (1993) Polymers near surfaces. Singapore, World Scientific

24. Qui LY, Bae YH (2006) Pharm Res 23:1

25. Wiltshire JT, Qiao GG (2007) Aust J Chem 60:699

26. Gao H (2012) Macromol Rapid Commun 33:722

27. Blencowe A, Tan JF, Goh TK, Qiao GG (2009) Polymer 50:5

28. Likos CN (2006) Soft Matter 2:478

29. Mathis C, Schmaltz B, Brinkmann M (2006) C R Chim 9:10751084

30. Wolkmuth WD, Duke T, Austin RH, Cox E (1995) Proc Natl Acad Sci USA 92:6887

31. Heuer DM, Saha S, Archer LA (2003) Electrophoresis 24:3314

32. Romiszowski P, Sikorski A (2006) J Mathem Chem 40(3):295

33. Sikorski A, Romiszowski P (2007) J Phys Condens Matter 19: 205136

34. Romiszowski P, Sikorski A (2009) J Mol Model 15:681-686

35. Račko D, Cifra P (2013) J Chem Phys 138:184904

36. Klymko K, Cacciuto A (2011) Phys Rev Lett 107:278302

37. Milchev A, Muller M, Klushin L (2014) Macromolecules 47(6): 2156-2168
38. Paturej J, Milchev A, Egorov SA, Binder K (2013) Macromolecules 46(19):8009

39. Gay C, de Gennes P-G, Raphael E, Brochard-Wyart F (1989) Branched polymers inside nanoscale pores. In: Kasianowicz JJ, Kellermayer MSZ, Deamer DW (eds) Structure and dynamics of confined polymers. NATO Series 3. High Technology, vol 87. Springer, Dordrecht, p 131

40. Wood JB (2004) J Appl Anim Welf Sci 7(2):95-106 http://www. thecephalopodpage.org/

41. Weeks JD, Chandler D, Andersen HC (1971) J Chem Phys 54:5237

42. Arnold A, Jun S (2007) Phys Rev E 76:031901

43. Jung Y, Ha B-Y (2010) Phys Rev E 82:051926

44. Jung Y, Kim J, Jun S, Ha B-J (2012) Macromolecules 45:32563262

45. Limbach H-J, Arnold A, Mann BA, Holm C (2006) Comput Phys Commun 174:704

46. Šolc K (1977) Polym News 4:67

47. Saha S, Heuer DM, Archer LA (2006) Electrophoresis 27:3181

48. Cifra P, Bleha T (2012) Soft Matter 8:9022

49. H. Nakanishi (1987) J Phys B 979-984

50. Schaefer DW, Joanny JF, Pincus P (1980) Macromolecules 13:1280

51. Sheng J (2012) Kaifu Luo. Soft Matter 8:367-374

52. Levy SL, Mannion JT, Cheng J, Reccius CH, Craighead HG (2008) Nano Lett 8:3839-3844

53. Burkhardt TW (1997) J Phys A: Math Gen 30:L167

54. de Gennes P-G (1979) Scaling concepts in polymer physics. Cornell University Press, Ithaca, NY

55. Milner ST, McLeisch TCB (1997) Macromolecules 30:2159-2166

56. Kramers HA (1940) Phys Utrecht 7:284

57. Minina E, Arnold A (2014) Soft Matter 10:5836

58. Polson JM, Montgomery LG (2014) J Chem Phys 141:164902 